\title{
LA APLICACIÓN DEL COACHING EN LOS PROCESOS DE APRENDIZAJE
}

\author{
Martínez Carballo, Manuel; Guillén Solórzano, Eduardo \\ Universidade da Coruña, Escuela Universitaria de Diseño Industrial
}

\section{RESUMEN}

El presente trabajo analiza la aplicación de las metodologías de coaching al proceso de aprendizaje, resaltando el enorme impacto que tiene en la motivación del alumnado y en los resultados conseguidos. Para ello se analiza su aplicación en el proceso de aprendizaje de alumnos del Grado en Ingeniería en Diseño y Desarrollo de Producto, especialmente en los trabajos académicos que realizan durante la carrera, relatando algunos ejemplos de la metodología y un caso práctico.

PALABRAS CLAVE Coaching, Aprendizaje, Empowerment

\section{CITA RECOMENDADA:}

Martínez Carballo, M., Guillén Solórzano, E. (2018). La aplicación del coaching en los procesos de aprendizaje. En E. de la Torre Fernández (ed.) (2018). Contextos universitarios transformadores: retos e ideas innovadoras. II Xornadas de Innovación Docente. Cufie. Universidade da Coruña (pág. 285-318). DOI capítulo: https://doi.org/10.17979/spudc.9788497496780.285 DOl libro: https://doi.org/10.17979/spudc. 9788497496780 


\section{ABSTRACT}

This paper analyses the implementation of coaching techniques to the learning process, highlighting the tremendous impact that may have in the attitude and motivation of the students as well as in the results obtained. We present the application of the methodology to the students of the Diploma in Design and Product Development Engineering, and especially to the academic essays, they develop during their careers, providing some examples of these techniques and one case study.

KEY WORDS: Coaching, Learning by doing, Empowerment 


\section{INTRODUCCIÓN. EL COACHING Y SUS ORÍGENES}

En este apartado se va a recoger una primera aproximación al concepto de "coaching" y sus orígenes de cara a entender en qué consiste la aplicación de esta disciplina. La palabra coaching tiene sus orígenes en el Siglo XV en la ciudad húngara de Kocs situada aproximadamente a unos 70 kilómetros de Budapest. Kocs era una población/ciudad de parada obligatoria para los viajeros que iban de Viena a Pest y que ha dado origen al nombre de un carruaje particular denominado "kocsi" que posteriormente, se tradujo al alemán como "kutsche", al italiano como "cocchio", al español como "coche" y al francés como "coach". Por lo tanto, etimológicamente el término coaching que, en la actualidad utilizamos de forma tradicional para referirnos a un proceso de desarrollo personal y profesional, proviene de la palabra francesa "coach" con el significado de un vehículo para transportar personas de un lugar a otro, es decir, del lugar de donde están a donde quieren ir. En este contexto, el conductor del carro (o "coach"), sirve de guía para el proceso de coaching facilitando el recorrido del cliente (o "coachee") a lo largo de su trayecto.

Por otra parte, son varios los autores que coinciden en señalar el origen del coaching en la mitología griega atribuyéndoselo a Sócrates en sus diálogos con Platón (Siglo IV a.C.) en donde parece ser que utilizaba la técnica de interrogar a una persona sobre algo y luego se procede a debatir la respuesta con el objetivo de llegar a tomar conciencia sobre el tema tratado (principal base del coaching en la actualidad).

En cualquier caso, dependiendo de cómo queramos explicarlo, podríamos decir que el coaching viene de uno u otro sitio en la historia, pero no fue hasta finales del siglo pasado cuando se empezó a utilizar en el ámbito organizacional. Inicialmente se aplica en Estados Unidos como un proceso de apoyo en el entrenamiento a deportistas de élite y sus resultados positivos fomentaron su aplicación en empresarios y directivos.

Partiendo de la idea de "un vehículo para transportar personas de un lugar a otro" y de la realización de preguntas por parte de Sócrates para ayudar a otros a que consiguiesen sus propios objetivos, podemos construir una definición actual de coaching como un proceso, 
dinámico, fluido y continuo; y como una relación existente entre dos o más personas en la que una de ellas (coach) acompaña a la otra (coachee) en un proceso para alcanzar las metas y objetivos planteados por el coachee, proceso que comienzan juntos con un acompañamiento a través de un rol de facilitador para encontrar las posibilidades, tanto reales como potenciales de que el coachee logre alcanzar el éxito deseado.

El coaching es una herramienta que ayuda a los líderes a mejorar sus capacidades de trabajo en equipo, además de aumentar su influencia sobre sus subordinados a la vez que crece el carisma y la empatía. En el ámbito organizacional lo podemos definir como un concepto impulsor, como una aplicación interpersonal, coaching ejecutivo, con el objetivo principal de alcanzar la mejora del desempeño y, a su vez, repercutir en el rendimiento de un directivo o de cualquier trabajador en su puesto de trabajo.

A continuación, se enumeran algunas definiciones utilizadas por profesionales de esta disciplina:

La Internacional Coaching Federation (ICF) agrupa la mayor organización de coaches profesionales a nivel mundial con casi 30000 coaches repartidos entre 143 países y más de dos décadas de trayectoria en la promoción de los más altos y exigentes estándares éticos y de calidad en la profesión de coaching (www.icf-es.com). Según esta entidad el coaching consiste en "una relación profesional continuada que ayuda a obtener resultados extraordinarios en la vida, profesión, empresa o negocios de las personas. Mediante este concepto el cliente profundiza en su conocimiento, aumenta su rendimiento y mejora su calidad de vida".

La International Coaching Comunity (ICC) fundada en 2001 y que en la actualidad cuenta con más de 12000 coaches certificados y con representación en 67 países de los 5 continentes (http://internationalcoachingcommunity.com/es) indican que "la esencia del coaching es ayudar a la persona a cambiar en la forma que ella desea y ayudarle a dirigirse en la dirección que quiere tomar. El Coaching apoya a la persona en todos los niveles, a convertirse en quien desea ser, y a convertirse en lo mejor que pueda ser". 
0'Connor, J. y Lages, A. (2005), fundadores de ICC, comentan que "el coaching es un juego de apuestas altas: nada menos que la felicidad. La gente acude al coaching porque quiere ser feliz o más feliz". El coaching no es una terapia, donde el psicólogo trate a su paciente, ni tampoco se trata de un proceso de "mentoring" donde el mentor nos orienta sobre qué camino tomar (Valderrama, 2009).

Timothy Gallwey, W. (2010), en los años 70, habla en su libro "El Juego Interior del Tenis" de que "siempre hay un juego interior que se está jugando en tu mente, independientemente del juego exterior que estás jugando. De cómo te comportes en este juego interior, dependerá el éxito 0 el fracaso de tu juego exterior".

Withmore, J. (2011) indica que "el coaching consiste en liberar el potencial de una persona para incrementar al máximo su desempeño. Consiste en ayudarle a aprender en lugar de enseñarle".

Miedaner, T. (2016) reflexiona que "el coaching cubre el vacío existente entre lo que eres ahora y lo que deseas ser. Es una relación profesional con otra persona que aceptará sólo lo mejor de ti y te aconsejará, guiará y estimulará para que vayas más allá de las limitaciones que te impones a ti mismo y realices tu pleno potencial".

Por último, indicar que según Dilts, R. (2004) el Coaching es una herramienta para el cambio y el coach es un facilitador de procesos de cambios en las personas. En este proceso facilitador el coach, no opina, no da soluciones y no da respuestas resultando ser un facilitador pedagógico del proceso de cambio en la educación. En el proceso de coaching es el cliente el que decide que camino va a tomar y que proceso de trabajo va a diseñar y cuáles van a ser sus objetivos $y / 0$ metas que quiere alcanzar.

\section{TIPOLOGÍAS DE COACHING}

Una vez abordado el origen del coaching y en qué consiste esta disciplina, conviene hacer una pequeña y breve clasificación de los tipos de coaching. En este sentido, indicar que existen múltiples clasificaciones y que una categorización no es excluyente de la otra. 
En primer lugar, cabe hacer la distinción entre el coaching individual como un proceso de acompañamiento individual a la persona frente al coaching de equipos para acompañar el desarrollo de equipos de trabajo. Ambas modalidades están referidas más en el ámbito organizacional.

En este ámbito organizacional también podemos hablar del coaching ejecutivo como un proceso de coaching individual dirigido a personas que "sus decisiones son estratégicas y tienen impacto en todo el sistema y, en última instancia se encuentran solas a la hora de tomarlas" (Viviane, 2014).

El coaching personal o life coaching hace referencia al coaching de habilidades para la vida diaria en el que se persigue el bienestar de la persona en los diferentes ámbitos de la vida.

También están el coaching deportivo que trabaja principalmente la motivación y el desarrollo de todo el potencial del deportista o de un equipo deportivo; el coaching educativo aplicado en el ámbito de la enseñanza: alumnos, padres, claustro,... ; entre otras modalidades del coaching. Sobre el coaching educativo se particularizará más en el siguiente apartado.

\section{EL COACHING EDUCATIVO}

Dentro de las múltiples referencias históricas que existen sobre el origen del coaching, tal y como se ha comentado anteriormente, también podemos encontrar una en el ámbito educativo. Fue en la Universidad de Oxford en el Siglo XIX cuando este vocablo cobró una nueva interpretación pasando a designarse en el argot de los estudiantes, a la persona que les ayudaba a identificar sus puntos débiles para superar los exámenes. En cualquier caso, y sea el ámbito que sea, estamos hablando de aquellas personas que acompañan a otras para alcanzar sus metas, potenciando sus habilidades y dando recursos para superar sus limitaciones.

En el contexto del coaching educativo uno de los cambios más importantes que puede aportar esta disciplina en el aula es que esta metodología no consiste en enseñar de forma directiva, sino que permite crear las condiciones propias para aprender y crecer, es decir, el coaching 
educativo no es directivo. También podemos hablar del coaching educativo a nivel de la dirección del centro para conseguir dotar a los directivos de herramientas ejecutivas. Y, un último nivel, sería el referido para docentes y claustro de profesores de cara a trabajar sobre el proceso de enseñanza, el trabajo de equipo, adquirir habilidades de comunicación, etc.

El coaching educativo ofrece una manera distinta de aprender buscando la mejora del rendimiento de forma individual, en contraposición al método de enseñanza tradicional que se les enseña lo mismo a todos los alumnos sin tener en cuenta sus habilidades y puntos fuertes limitando enormemente el potencial y creatividad (aprendizaje integral del alumno). Para ello, se necesita un cambio de rol por parte del profesor que pasaría a un segundo plano y se convertiría en un guía en el proceso de aprendizaje. Así, el alumno se convierte entonces en el protagonista del aprendizaje, y los contenidos se van descubriendo conjuntamente. Por otra parte, también es recomendable que el profesor cree espacios donde trabajar otros aspectos que van más allá de los contenidos y que son fundamentales para el desarrollo de los alumnos.

Existen 5 claves o principios básicos del coaching educativo que es necesario conocer y tener en cuenta para su aplicación.

1.- Una de las principales claves del coaching educativo no está en enseñar de forma directiva a todos los alumnos por igual sino en encontrar y desarrollar el verdadero potencial de cada uno de los alumnos.

2.- Ser consciente del potencial de uno mismo y adoptar una actitud no directiva y abierta al cambio en la relación de aprendizaje que permita la reflexión, la observación, etc., en el aula 3.- El empoderamiento de los alumnos surge de una educación no del conocimiento sino de la responsabilidad que implica este conocimiento. En este sentido, el coaching educativo es una herramienta muy valiosa para empoderar a los alumnos y hacerlos más independientes.

4.- El feedback es una herramienta del coaching que también está presente en el caso del coaching educativo para poder maximizar el aprendizaje del alumno. 
5.- La aplicación del coaching educativo en el aula sólo será viable cuando se potencie el desarrollo de distintas competencias, tales como, la escucha activa, la inteligencia emocional, la paciencia, la atención, entre otras.

\section{DESCRIPCIÓN DE LA EXPERIENCIA}

Una vez contextualizado el proceso de coaching en el ámbito educativo, se ha planteado la aplicación de este nuevo enfoque a los trabajos desarrollados por parte de los alumnos en el contexto de algunas asignaturas del Plan de Estudios de la titulación de Grado en Ingeniería en Diseño y Desarrollo de Producto, y en particular en la materia que lleva por nombre Administración y Organización Industrial.

En este apartado se detalla el enfoque anterior a esta aplicación, y el nuevo enfoque tras la aplicación de metodologías propias del coaching.

\section{Situación de partida}

Generalmente los trabajos académicos que se venían planteando en la asignatura consistían en la propuesta de elaboración de un plan de industrialización de un producto conocido, a propuesta generalmente del docente. En este caso, se escogía de entre un grupo de productos industriales preseleccionado alguna alternativa, para que los alumnos elaboraran un Plan de Industrialización aproximado a ese producto, partiendo siempre de unos volúmenes de producción predeterminados, establecidos por parte del profesor.

Generalmente los alumnos recibían esta tarea como algo aplicado, fundamentado en los conceptos más teóricos vistos en clase, pero sin una mayor utilidad que la puesta en práctica de los mismos, por lo que generalmente el nivel de motivación y de entusiasmo, y consecuentemente el esfuerzo a realizar durante el curso, estaban condicionados por esta situación de partida. 
Tras la decisión de comenzar a aplicar las metodologías de coaching en el aula, como experiencia innovadora, la situación se revierte totalmente. A continuación, se detallan los pasos seguidos con el nuevo enfoque.

\section{Primero: Contextualización de la Experiencia}

En un primer momento, y coincidiendo con las presentaciones de las asignaturas a inicio de curso, se les plantea la Guía Docente de la materia, bajo las mismas condiciones que en el anterior enfoque. Si bien, se comienza a abundar en la oportunidad que se les brinda a la hora de hacer el trabajo de la asignatura, que sigue consistiendo en la elaboración de un Plan de Industrialización. La única diferencia es que, en este caso, el docente, más allá de aplicar un enfoque ejecutivo y directivo sobre lo que se ha de trabajar, les brinda la oportunidad de que ellos escojan un producto más o menos innovador, que enlace con sus intereses, con sus objetivos o con aquellos sectores en los que les gustaría desarrollar su carrera profesional. Este cambio de contenidos, aparentemente surte una mayor motivación en el alumnado, pues pasa de resultar "un encargo", para cambiar a "una oportunidad". De alguna manera este "empoderamiento" del alumno, a la hora de determinar y elegir sus tareas, y la mayor responsabilidad que se les exige, resulta un factor de motivación importante, pues aún a sabiendas de que el trabajo a realizar será mayor, resulta que el entusiasmo con el que reciben el encargo también resulta mucho mayor.

\section{Segundo. Establecimiento de Objetivos}

Generalmente las primeras tutorías que se realizan con los equipos de trabajo se centran en el establecimiento de objetivos, tanto académicos de la asignatura, como personales de cada alumno. Cuando se les pregunta por sus objetivos profesionales a corto plazo, y sobre los académicos en la asignatura, resulta chocante en un primer momento para ellos. Se trata pues de las primeras "preguntas poderosas" con las que el alumno se encuentra, a pocos meses de finalizar sus carreras (pues se trata de una asignatura de último curso), en pocas ocasiones se 
habían planteado durante la misma qué irían a hacer al finalizar. Se observa que, en muchos casos, el proceso de aprendizaje del alumno siempre ha estado guiado por el sistema educativo, por su entorno social, o incluso familiar, y hasta casi los últimos cursos han avanzado "de la mano" del sistema, sin haber tenido ocasión de tomar demasiadas decisiones. Es ahora cuando comienza a asomarse al abismo que supone finalizar la etapa de formación y plantearse el inicio de una carrera profesional, y quizás por el contexto económico y social actual, muchos se encuentran perdidos en esta decisión. Así pues, estas primeras tutorías en equipos de trabajo resultan tremendamente interesantes a la hora de que ellos reflexionen sobre dichos objetivos tanto personales, como académicos y profesionales, y que perciban la oportunidad de hacer algo práctico y de utilidad que les aporte valor en ese camino que pronto comenzarán a andar.

Tras las dos primeras tutorías grupales, se establecen por lo tanto dichos objetivos en el equipo, y se procura definir un producto que resulte motivante, interesante, y que pueda abrirles puertas en un futuro próximo en el mercado laboral. Es ahí cuando encuentran ese vínculo entre sus objetivos, sus valores, y el plan de acción que se sucederá en las próximas semanas.

\section{Tercero: Establecimiento del Plan de Acción}

Generalmente tras la determinación de los objetivos, el equipo se decanta por algún producto innovador que a pesar de no existir y de tener que desarrollarlo conceptualmente y a través de planos constructivos y modelos en 3D, resulta más motivante que cualquier producto ya existente. Es a partir de ahí, cuando se establecen las diferentes etapas y entregas del Plan de Industrialización, cuyos avances serán supervisados de semana en semana en nuevas tutorías. Si bien, esta segunda fase del trabajo es algo más directiva, pues efectivamente se corresponde con la parte académica de la asignatura en cuestión. Sin embargo, existe toda una actividad complementaria en la que se procura que el alumno vaya estableciendo acciones y actividades que procuren una mayor visibilidad del trabajo realizado, poniendo en valor todo 
el esfuerzo que se va realizando. Así pues, y según cuales hayan sido los objetivos de partida, el docente-coach persigue que en cada tutoría, además de las actividades académicas a realizar, se establezcan otras actividades 0 tareas complementarias para valorizar dicho esfuerzo, y así aproximarse poco a poco a los objetivos previamente descritos.

\section{Cuarto: Seguimiento}

El transcurso de las tutorías sucesivas, se centra complementariamente entonces en dos frentes, por un lado, el avance del proyecto académico en cuestión, y en segundo lugar en la aproximación al objetivo previamente establecido del proyecto. Es en estas tutorías semanales donde el docente coach, va intentando resolver las dificultades existentes con cada equipo, tratando de que ellos mismos sean quienes encuentren las soluciones y los recursos necesarios, procurando en todo momento el desarrollo de esas habilidades y competencias tan buscadas, y que se centran en el trabajo en equipo, la motivación, el liderazgo, la resolución de conflictos, creatividad, y un largo etcétera que tan necesarias son hoy en día para abordar con garantías cualquier profesión. Durante esta etapa, se cuestiona continuamente al alumno sobre su estado de ánimo, se celebran los pequeños éxitos conseguidos durante el proceso, y se procuran soluciones continuamente a las dificultades que van surgiendo. Bien es cierto que esta labor de coaching, en muchas ocasiones también se complementa por parte del docente con sesiones de mentorización y asesoramiento, pues habitualmente también se comparten experiencias profesionales del mismo con los alumnos para que puedan contar con referentes pasados.

\section{Quinto: Cierre}

El transcurso del proceso de coaching continúa durante los meses que dura el cuatrimestre en el que se enmarca la asignatura, por lo que es una etapa más o menos prolongada de tiempo, en el que el equipo, y cada uno de los alumnos, se van percatando de que esos objetivos que pueden aflorar cuando uno realmente se lo plantea, finalmente pueden ser alcanzados a base 
de la planificación detallada de estos planes de acción, y de la fuerza y energía suficientes como para perseverar en ello. Generalmente este enfoque en el que el trabajo a realizar se acompaña de sesiones de coaching, o en general del abordaje de un proceso de coaching grupal, la dedicación del alumno es mucho más intensa y gratificante para todos, lo que resulta en un efecto tremendamente positivo para el grupo y, por ende, para el docente. Finalmente, y según cuales sean los resultados alcanzados, se procura también algún tipo de incentivo para los alumnos tales como la solicitud de algún registro industrial de protección de sus resultados, vía la propiedad industrial del mismo, y vía la propiedad intelectual según sean Ios casos. Este "premio" o incentivo es también recibido como algo muy positivo por parte de Ios alumnos, ya que generalmente resulta un nuevo refuerzo contingente a su labor. Sin embargo, más allá de estos incentivos, y de las calificaciones obtenidas en los trabajos, el mayor premio o estímulo para ellos es el haberse sentido parcialmente responsables de su proceso de aprendizaje, habiendo tomado desde el primer momento las riendas de su trabajo, y de los esfuerzos a realizar, y habiendo desarrollado durante todo el semestre las habilidades y competencias necesarias para alcanzar el éxito.

\section{RESULTADOS}

En este apartado se comenta como ejemplo de los resultados obtenidos a la hora de aplicar este nuevo enfoque, un caso práctico abordado en el curso académico 2014/15, y que por la repercusión mediática que tuvo, supuso un hito y un referente para este nuevo enfoque donde Coaching y Docencia se combinan en igual medida. Se trata del proyecto Dronlife.

En el curso académico 2014/15, se planteó tal y como se detallaba en el apartado anterior la metodología de trabajo a seguir en la asignatura de Administración y Organización Industrial de $4^{0}$ curso, donde uno de los grupos de alumnos (en este caso compuesto por 4 alumnas), decidieron apostar por elaborar un Plan de Industrialización de un Dron que pudiera transportar órganos para trasplantes. 
En las sesiones de selección de propuestas, y aún a sabiendas de que tendrían que abordar toda la parte de diseño y desarrollo de un producto inexistente, sintieron motivación por trabajar en ideas que estuvieran llamadas a convertirse en tendencia en los próximos años. De acuerdo con el ránking de Harvard que se publica todos los años sobre las tecnologías con más potencial de desarrollo, y la agenda $\mathrm{H} 2020$ con los retos de la sociedad para estos próximos años, lograron unir las tecnologías de los Drones (que entonces estaban despegando), con uno de los retos de la sociedad que a todos nos preocupa y que resulta del ámbito de la salud. Así pues, buscaron la manera de combinar ambos enfoques, y finalmente dieron con la idea de desarrollar un dron que pudiera acortar tiempos en los procesos de trasplante de órganos.

En estas primeras tutorías se procuró por parte del docente que esta idea estuviera vinculada intensamente con los objetivos personales de cada una de ellas, y con los del equipo en global, lo que resultaba evidente por los valores de solidaridad y de innovación que manifestaban tener todas ellas. Así pues, se tomó la decisión, y se comenzó a trabajar en la propuesta.

Siendo este el punto de partida, el plan de acción se desarrolló a través de las diferentes etapas que se recogían en el Plan de Industrialización que tenían que elaborar, y que se expone a continuación:

- Conceptualización de producto

- Definición del Producto-Mercado

- Desarrollo de la propuesta

- Modelización 3D

- Planos constructivos

- Hoja de Escandallo y propuesta de componentes y materiales

- Mapa de Procesos Industriales para la Fabricación

- Determinación de la demanda prevista y precisión del volumen de producción

- Fichas de procesos 
- Cálculo de costes

- Dimensionamiento de la planta industrial, y distribución interna de la misma

- Diseño del Flujo de materiales

- Maestro de Producción

- Maestro de Aprovisionamientos

- Planillas de tiempo para la plantilla

Cada uno de estos ítems suponía un entregable a revisar cada una de las semanas del curso, hasta su entrega final, y constituían el cuerpo de contenidos del Plan de Industrialización. Siendo ya de por sí una carga de tareas y trabajos intensa, a repartir entre los cuatro o cinco integrantes de cada grupo, el equipo en cuestión se planteó como reto personal la valorización de todo este esfuerzo a través de su presentación a un concurso en el que casualmente estaban buscando aplicaciones civiles para los drones (algo que en aquél entonces era innovador, pues los drones habían estado históricamente destinados a aplicaciones militares). Se trataba del concurso internacional Drones For Good, convocado por la Oficina del Primer Ministro y Jeque de Dubai, y dotado con un premio de 1 millón de dólares.

Tuvieron la fortuna de que, tras la presentación de un video de animación con una duración escasa de 1 minuto, fueron preseleccionadas entre más de 800 proyectos de hasta 62 países diferentes, entre las 20 mejores propuestas del mundo, y por ello, fueron invitadas a la fase de demostración de dichas tecnologías al evento que se celebraría en Febrero de 2014 en Dubai, donde los veinte equipos preseleccionados competirían por ese premio de 1 millón de dólares a la idea más innovadora.

Lo cierto es que, en ese primer momento, resultó tremendamente difícil que nadie creyera lo que estaba pasando, recibiendo numerosas muestras de desconfianza y de escasa credibilidad a lo que se les estaban planteando desde Emiratos Árabes Unidos. Ni el premio, ni la convocatoria, ni la invitación a participar en el concurso resultaban creíbles para nadie del entorno en el que se encontraban, lo que llegó incluso a hacer dudar a las propias interesadas 
sobre la realidad de todo aquello, más aún cuando les daban un plazo de tiempo de escasos días para confirmar si estarían en condiciones de participar en dicho concurso.

Por fortuna, y en una sesión de coaching intensa, logramos visibilizar el momento, encontrar la energía necesaria, y apostar por un SI. Fue entonces cuando dio comienzo toda una carrera contra reloj, pues había que estar en Dubai con un Dron que volase, que pudiera transportar una nevera con un órgano, y que además se pareciera algo a lo que se había enviado como animación.... Pues hasta ese momento... no había nada desarrollado.

Igualmente se necesitaba contar con apoyos financieros para sufragar los costes de tal expedición, pues si bien las interesadas estaban invitadas a participar con todos los gastos pagados, no era así el caso de los acompañantes que tuvimos ocasión de participar también en la aventura, así como el piloto que finalmente se desplazó a Dubai para la demostración de vuelo.

Habiendo ocurrido esto en las primeras semanas de octubre, quedaban tan solo cuatro meses para tener todo listo para el momento, y no era poco lo que había que conseguir...

- Financiación

- Acuerdo tecnológico con fabricante de drones

- Licencias de vuelo

- Permisos y trámites ante la Agencia de Aviación Civil de Emiratos Árabes Unidos

- Visados

- Construcción de la maqueta de la nevera

- Preparación de las presentaciones

- Elaboración de material promocional

- Diseño y construcción del stand corporativo

Cada semana afloraban nuevas dificultades, y cada semana procurábamos nuevas soluciones cada vez más ingeniosas y creativas para mantener el ritmo de trabajo que nos trasladaban desde Dubai... Era precisamente en estas reuniones donde cobraba especial relevancia todo el proceso de Coaching, pues más allá de adoptar un enfoque directivo y ejecutivo por parte del 
docente, se procuraba dar espacio suficiente a la toma de decisiones por parte del equipo... pues a la postre, se trataba de su proyecto, y de sus objetivos.

Así, en esta labor de acompañamiento, conjugando diferentes herramientas propias de la metodología del coaching, se iban dando pequeños pasos que iban aproximando poco a poco Ios resultados al objetivo pretendido.... Hasta que finalmente... llegó el gran día... hacer las maletas, y emprender rumbo a Dubai.

Todo lo que allí aconteció, lo fueron relatando puntualmente los medios de comunicación, pues se coordinó toda una campaña con medios locales y nacionales de prensa, radio y televisión, así como también se combinaba la campaña con toda la repercusión mediática que ya de por sí tenía el concurso en Dubai, y que retransmitían numerosas cadenas internacionales, desde Discovery Channel, hasta la BBC.

Aquellos días, entre pruebas, ensayos y preparativos, todo discurrió con un total frenesí, hasta el día de la gran final. Tras pasar la primera eliminatoria, y quedar entre los 10 proyectos mejor valorados, finalmente se consiguió un bien merecido tercer puesto, tras vencer al proyecto del MIT, y a otro de la Universidad de Oxford, y quedar entre los tres primeros con un proyecto privado de una empresa tecnológica alemana, y otro de Suiza, finalmente ganador de aquella edición.

Los días posteriores al concurso si cabe fueron todavía más intensos, pues recibimos una invitación personal del Jeque de Dubai para prolongar la estancia durante una semana más, y así poder participar en la Cumbre de Gobiernos de la Región precisamente dedicada aquél año a la innovación tecnológica. Fue allí donde embajadas y comitivas de hasta 70 países, muchos de ellos de la región, conocieron de primera mano las propuestas de los 10 finalistas, interesándose muchos por las tecnologías que allí se presentaban. 


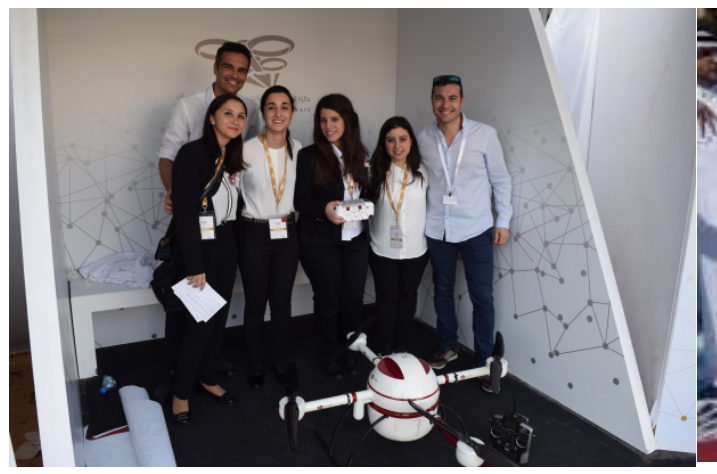

Imagen 1: Equipo Dronlife durante el concurso

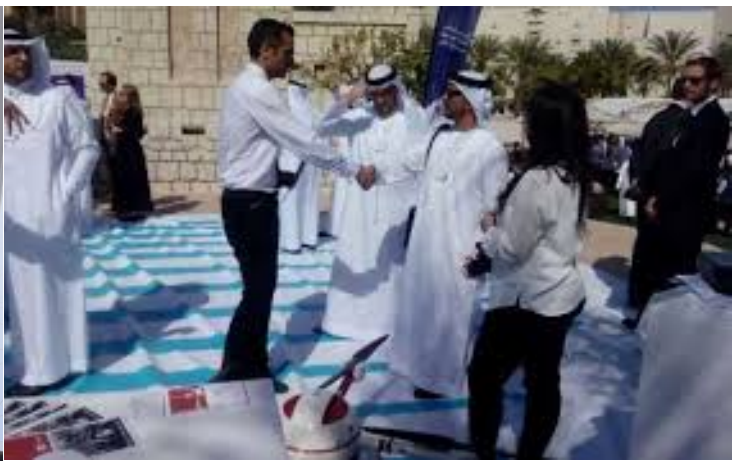

Imagen 2: Comitivas durante la Cumbre de Gobiernos

El retorno a España fue igualmente espectacular.... Fueron semanas de presentaciones, asistencias a actos y eventos, participaciones en conferencias y congresos de todo tipo, donde el proyecto de Dronlife iba consiguiendo despertar cada vez un mayor interés. Prueba de ello son los más de 150 impactos mediáticos en prensa, radio y televisión que al cabo de un año logro alcanzar el proyecto, y que se recogen en las referencias de este artículo.

\section{CONCLUSIONES}

Como conclusiones a la hora de valorar la aplicación de procesos de coaching en el ámbito educativo, generalmente podemos resaltar que los efectos positivos son enormes si el docente es capaz de complementar el esfuerzo académico que debe realizar el alumno para asimilar conceptos y técnicas concretas, con los valores y objetivos que ese alumno pueda precisar de cara a un futuro más o menos cercano. Es bastante frecuente que el alumno se encuentre desmotivado en el aula por no ver la conexión de lo que allí hace con lo que realmente él desea alcanzar, y por ello debiera de reflexionarse sobre esa conexión. Si además, somos capaces de que el alumno vincule esos objetivos con sus valores más profundos como persona, entonces la fuerza que cobra dicho objetivo es tremenda, y la predisposición al trabajo, al esfuerzo y al sacrificio que debe hacer, aumenta considerablemente. 
El caso de Dronlife resultó extraordinario por la repercusión que alcanzó el proyecto, pero son muchos otros los ejemplos que en mayor o menor medida se pone de manifiesto la utilidad del coaching educativo para alcanzar un mejor rendimiento por parte del alumno, tanto en lo que se refiere a las actividades vinculadas propiamente a las asignaturas, como también en lo relativo al desarrollo de habilidades y competencias básicas que tan necesarias son hoy en día.

Merece también especial mención los resultados positivos que brinda al docente, pues siempre que mejora el ambiente en el aula, el esfuerzo por parte de los alumnos y los resultados conseguidos, el docente recibe su premio en condición de responsable en última instancia del aprendizaje logrado en el aula, siendo co-responsable en todo caso de dichos éxitos, y aumentando la motivación y la predisposición de cara a nuevos retos profesionales.

\section{REFERENCIAS}

- Cobo, J.M. (2000). Aprendiendo de la experiencia. Capital Humano, núm. 136, pp. 68-71

- Dilts, R. (2004). Coaching. Herramientas para el cambio. Barcelona. Urano.

- Goleman, D. (2000). Ledership That Gets Results. Harvard Business Review, marzoabril, pp. 78-92.

- Miedaner, T. (2000). Coaching para el éxito. Conviértete en el entrenador de tu vida personal y profesional. Barcelona: Urano.

- O’Connor, J. y Lages, A. (2005). Coaching con PNL. Guía práctica para obtener 10 mejor de ti mismo y de los demás. Barcelona, Urano.

- Timothy Gallwey, W. (2010). El juego interior del tenis. Editorial Sirio.

- Valderrama, B. (2009). Desarrollo de competencias de Mentoring y Coaching. Madrid: Pearson Educación.

- Viviane, L. (2014). Coaching. Un camino hacia nuestros éxitos. Madrid: Empresa\&Gestión. 
- Whitmore, J. (2016). Coaching. El método para mejorar el rendimiento de las personas.

Barcelona: Paidós Empresa.

- Zeus, P. y Skiffington, S. (2002). Guía completa de coaching en el trabajo. Madrid: McGraw Hill.

\section{REFERENCIAS SOBRE EL PROYECTO DRONLIFE}

1. Autonomic media - La Voz de Galicia Deza - Tabeirós. Date: 22/10/2014. Language: Spanish

http://www.lavozdegalicia.es/noticia/deza/2014/10/22/talento-local-

mundo/0003_201410D22C119968.htm?utm_source=facebook\&utm_medium=refe rral\&utm_campaign =fbdeza

Dronlife goes to The Drones for Good Award semifinals. First steps.

2. Autonomic media - El Faro de Vigo Deza - Tabeirós - Montes. Date: 26/10/2014.

Language: Spanish

http://www.farodevigo.es/portada-deza-tabeiros-montes/2014/10/26/andrea-maganidea-reducir-tiempo/1119168.html

Dronlife goes to The Drones for Good Award seminfinals. Summary about the project and first steps.

3. Autonomic media - El Correo Gallego. Date: 07/11/2014. Language: Spanish http://www.elcorreogallego.es/galicia/ecg/busca-un-dron-salvavidas/idEdicion-201411-07/idNoticia-899759/

Summary about the Dronlife project and The Drones for Good Award.

4. Autonomic media: Compostimes. Date: 12/11/2014. Language: Spanish http://compostimes.com/2014/11/el-grupo-innova-en-las-semifinales-del-concursointernacional-drones-forgood/?doing_wp_cron $=1415828356.0124130249023437500000$ 
Interview to the Innova team, Dronlife's project creator.

5. Local media: Ferrol 360. Date: 07/01/2015. Language: Spanish

http://www.ferrol360.es/estudiantes-de-diseno-industrial-de-ferrol-trabajan-paratrasladar-organos-en-drones/

Iffe Business Group promotes Dronlife project.

6. National media: ABC. Date: 07/01/2015. Language: Spanish

http://www.abc.es/agencias/noticia.asp?noticia $=1759814$

Iffe Business Group promotes Dronlife project.

7. Autonomic media: El Ideal Gallego. Date: 07/01/2015. Language: Spanish

http://www.elidealgallego.com/articulo/coruna/empresa-corunesa-entra-sector-civilaviones-no-tripulados/20150107170752224447.html

Iffe Business Group promotes Dronlife project.

8. Autonomic media: El Correo Gallego. Date: 08/01/2015. Language: Spanish

http://www.elcorreogallego.es/galicia/ecg/iffe-financia-proyecto-un-drontrasplantes/idEdicion-2015-01-08/idNoticia-909519/

Iffe Business Group promotes Dronlife project.

9. Autonomic media: El Progreso. Date: 08/01/2015. Language: Spanish

$\underline{\text { PDF }}$

Iffe Business Group promotes Dronlife project.

10. Local media: La opinión Coruña. Date: 08/01/2015. Language: Spanish

http://www.laopinioncoruna.es/economia/2015/01/08/grupo-industrial-corunes-iffecrea/915579.html

Iffe Business Group promotes Dronlife project.

11. Autonomic media: La Voz de Galicia Empresas. Date: 11/01/2015. Language: Spanish

$\underline{\text { PDF }}$

Summary about the Dronlife project and the travel to Dubai to participate in The Drones for Good Award. 
12. National media: Daysign magazine. Date: $13 / 01 / 2015$. Language: Spanish http://www.daysignmagazine.com/\#!DRONLIFE-Semifinalista-del-ConcursoInternacional-Drones-for-Good-Ilevado-a-cabo-por-Ios-Emiratos-\%C3\%81rabesUnidos/c18up/976E0734-9BFC-40E3-9323-AE9971A9C0F7

Interview to the Innova team, Dronlife's project creator. Summary about the project and the steps to follow.

13. Autonomic media: La voz de Galicia. Date: 28/01/2015. Language: Spanish http://www.lavozdegalicia.es/noticia/sociedad/2015/01/28/estudiantes-gallegascrean-dron-transporta-organos-trasplantes/0003_201501G28P24991.htm Summary about the Dronlife project and the first flight test done.

14. Local media: Diario de Ferrol. Date: 26/01/2015. Language: Spanish http://www.diariodeferrol.com/articulo/ferrol/alumnas-campus-disenan-dronoptimizar-transporte-organos/20150125230737113332.html Summary about the Dronlife project and the travel to Dubai to participate in The Drones for Good Award.

15. Autonomic media: El Faro de Vigo. Date: 28/01/2015. Language: Spanish http://www.farodevigo.es/portada-deza-tabeiros-montes/2015/01/28/salvar-vidaspremio/1173474.html

Summary about the first flight test done and the travel to Dubai to participate in The Drones for Good Award

16. International media: The Gadget Website. Date: 28/01/2015. Language: English http://www.t3.com/news/drones-for-good-awards Summary about the Drones for Good Award.

17. National media: El Mundo. Date: 29/01/2015. Language: Spanish http://www.elmundo.es/economia/2015/01/26/54c6804dca47417a0d8b4582.html Summary about The Drones for Good Award and all the participants and presented projects. 
18. American Media: Forbes. Date: 31/01/2015. Language: English http://www.forbes.com/sites/jenniferhicks/2015/01/31/uae-opens-internationaldrones-for-good-competition/

Summary about The Drones for Good Award and the presented projects

19. Autonomic Media: El correo Gallego. Date:01/02/2015. Language: Spanish http://www.elcorreogallego.es/galicia/ecg/dron-salvavidas-ferrol-medira-proyectosnasa-dubai/idEdicion-2015-02-01/idNoticia-913783/\# Summary about what would be the Dronlife participation in The Drones for Good Award.

20. Autonomic media: El progreso. Date: 01/02/2015. Language: Spanish http://elprogreso.galiciae.com/noticia/345200/drones-para-salvar-vidas Summary about what would be the Dronlife participation in The Drones for Good Award.

21. Autonomic media: La voz de Galicia. Date: 01/02/2015. Language: Spanish http://www.lavozdegalicia.es/noticia/2015/02/01/drones-explotan-cielogallego/0003_201502G1P37991.htm The emerging drones field in Galicia. Pioneer companies, including Dronlife S.L.

22. Autonomic media: El Correo Gallego. Date: 01/02/2015. Language: Spanish http://www.elcorreogallego.es/galicia/ecg/unico-equipo-formado-estudiantes-100femenino/idEdicion-2015-02-01/idNoticia-913784/

Summary about all the steps done in the project before the date: deliveries for the award, financing, supports, ...

23. Media: Acceda Ortopedia (Pontevedra). Date: 02/02/2015. Language: Spanish http://www.accedaortopedia.es/noticias.aspx?id=17

Summary about Dronlife project and what would be the participation in The Drones for Good Award.

24. Autonomic media: El Correo Gallego. Date: 06/02/2015. Language: Spanish 
http://www.elcorreogallego.es/galicia/ecg/diario-un-dron-ferrolano-concursodubai/idEdicion-2015-02-06/idNoticia-914679/

Summary about the team first days in Dubái.

25. Emirates media: WAM News Agency. Date: 06/02/2015. Language: English http://www.wam.ae/en/news/emirates/1395276138787.html Contestants to the final round in The Drones for Good Award.

26. Emirates media: Khaleej Times. Date: 07/02/2015. Language: English http://www.khaleejtimes.com/nation/general/final-round-of-uae-drones-for-goodawards-today

Contestants to the final round in The Drones for Good Award.

27. Autonomic media: El Correo Gallego. Date: 07/02/2015. Language: Spanish http://www.elcorreogallego.es/galicia/ecg/cielo-alcance-dedos/idEdicion-2015-0207/idNoticia-914913/

Summary about the team stay in Dubai. Access to the final round.

28. Autonomic media: El Correo Gallego. Date: 07/02/2015. Language: Spanish http://www.elcorreogallego.es/galicia/ecg/dron-salvavidas-ferrol-pasa-gran-finaldubai-un-equipo-100-femenino/idEdicion-2015-02-07/idNoticia-914912/ Dronlife's access to the final round in The Drones for Good Award.

29. Emirates media: The Gulf Today. Date: 07/02/2015. Language: English http://gulftoday.ae/portal/bdee8720-accf-4c1a-9e3a-d7ee4da34000.aspx Summary about The Drones for Good Award and all the participants and presented projects.

30. Emirates media: Anbaanet. Date: 07/02/2015. Language: English http://www.anbaanet.com/feed/3886635.html?ref=archive Summary about the final round and the finalist in the different categories

31. Autonomic media: El Correo Gallego. Date: 08/02/2015. Language: Spanish 
http://www.elcorreogallego.es/galicia/ecg/gran-dron-salvavidas-ferrol-tres-mejoresmundo/idEdicion-2015-02-08/idNoticia-915022/

Summary about the final round and our third place in the award.

32. Autonomic media: La voz de Galicia. Date: 08/02/2015. Language: Spanish http://www.lavozdegalicia.es/noticia/tecnologia/2015/02/08/tercer-puesto-dubaigallegas-crearon-dron-trasplantes/0003_201502G8P31993.htm Summary about the final round and our third place in the award.

33. German media: Der-Spiegel. Date: 08/02/2015. Language: German $\underline{\text { PDF }}$

Summary about The Drones for Good Award

34. Autonomic media: El Correo Gallego. Date: 09/02/2015. Language: Spanish http://www.elcorreogallego.es/galicia/ecg/primer-ministro-emiratos-interesaproyecto-gallego/idEdicion-2015-02-09/idNoticia-915176/

Dronlife live experience in the final round in The Drones for Good Award and invitation to the Governmental Summit.

35. Brazilian media: BOL Notícias. Date: 09/02/2015. Language: Portuguese http://noticias.bol.uol.com.br/ultimas-noticias/tecnologia/2015/02/09/copa-dosdrones-e-vencida-por-robo-que-mantem-estabilidade-em-batidas.htm The Drones for Good Award final round

36. Media: Microdrones International. Date: 09/02/2015. Language: Spanish https://www.microdrones.com/en/news/detail/md4-1000-based-dronlife-nominatedfor-uae-drones-for-good-award/

Dronlife, based on a md4-1000, and its nomination for the $1^{\text {st }}$ UAE Drones for Good Award.

37. British media- International edition: CNN. Date 09/02/2015. Language: English http://edition.cnn.com/2015/02/09/tech/gimball-drones-for-good/ The Drones for Good Award finalists and winner. 
38. Local media: Diario de Ferrol. Date 09/02/2015. Language: Spanish http://www.diariodeferrol.com/articulo/ferrol/dron-ferrolano-fue-elegido-mejoresmundo/20150208211549114642.html Dronlife reaches the $3^{\text {rd }}$ place in the international category of the Drones for Good Award.

39. Autonomic media: El Correo Gallego. Date: 10/02/2015. Language: Spanish http://www.elcorreogallego.es/galicia/ecg/gran-dron-salvavidas-ferrol-exhibe-dubaigurus-microsoft-google/idEdicion-2015-02-10/idNoticia-915425/ Summary about the Dronlife's stay on the Governmental Summit.

40. Autonomic media: Faro de Vigo. Date: 10/02/2015. Language: Spanish http://www.farodevigo.es/portada-deza-tabeiros-montes/2015/02/10/dron-salvavidasestradense-andrea-magan/1181502.html Dronlife's $3^{\text {rd }}$ place in the Drones for Good Award.

41. Autonomic media: El Correo Gallego. Date: 11/02/2015. Language: Spanish http://www.elcorreogallego.es/galicia/ecg/dron-ferrol-atrae-interes-gigantesmundiales-innovacion/idEdicion-2015-02-11/idNoticia-915633/ Dronlife project on the Governmental Summit.

42. Autonomic media: La Región. Date: 11/02/2015. Language: Spanish http://www.laregion.es/opinion/carla.reyes.uschinsky/chicasdrone/20150211083220521692.html Recognition to the project creators.

43. Qatar media: QCRI. Date: 12/02/2015. Language: English http://qcri.org.qa/resources/in-the-media/uae-contest?backArt=1 Summary about The Drones for Good Award 44. Irish media: The Irish Time. Date: 12/02/2015. Language: English http://www.irishtimes.com/business/technology/robotics-groups-compete-for-1m-atdrones-for-good-in-dubai-1.2099892 
Summary about The Drones for Good Award

45. Media: Wamda, platform of programs and networks that aims to accelerate entrepreneurship ecosystems across MENA. Date: 12/02/2015. Language: English http://www.wamda.com/2015/02/bright-future-drones-industry-uae-contest Summary about The Drones for Good Award and its winners and participants.

46. Autonomic media: El correo gallego. Date: 13/02/2015. Language: Spanish http://www.elcorreogallego.es/galicia/ecg/exitoso-dronlife-alumnas-eudi-aterrizagalicia/idEdicion-2015-02-13/idNoticia-916012/

Dronlife team arrives to Spain after their participation in The Drones for Good Award.

47. Local media: Diario de Ferrol. Date: 14/02/2015. Language: Spanish http://www.diariodeferrol.com/articulo/ferrol/alumnas-diseno-industrial-regresancasa-exito-cosechado-dron-dubai/20150213221959115112.html Dronlife team arrives to Spain after their participation in The Drones for Good Award.

48. International media: Tech Republic. Date: 17/02/2015. Language: English http://www.techrepublic.com/pictures/photos-drones-for-good-from-disaster-rescueto-replanting-the-worlds-forests/3/

Summary about The Drones for Good Award and the presented projects.

49. Xunta de Galicia Igualdade. Date: 19/02/2015. Language: Galician/Spanish http://igualdade.xunta.es/gl/protagonistas/andrea-magan-susana-lopez-tays-ferrer-emacarena-arcay

Project Dronlife: their creators and participation in The Drones For Good Award.

50. British media: The Gadget Show. Date: 19/02/2015. Language: English http://gadgetshow.channel5.com/news/drones-rescue-meet-worlds-smartest-drones Summary about some of the projects which have participated in The Drones for Good Award.

51. Autonomic media: El Correo Gallego. Date: 20/02/2015. Language: Spanish 
http://www.elcorreogallego.es/galicia/ecg/liberia-quiere-dron-ferrol-vacunasebola/idEdicion-2015-02-20/idNoticia-917234/

Liberia wants to apply the Dronlife project to transport ebola vaccines

52. Emirates media: UAE interact. Date: 23/02/2015. Language: English http://www.uaeinteract.com/german/news/default.asp?ID =502 Drones legislation on the UAE and summary about The Drones for Good Award

53. Media: Microdrones Asia. Date: No date registered. Language: English http://www.cnuav.com/cms/index.php?a $=$ shows\&catid $=67 \&$ id $=48$ Dronlife, based on a md4-1000 and The Drones for Good Award

54. National media: Transplantes $n^{0} 20$. Date: February 2015. Language: Spanish http://issuu.com/medysalud/docs/trasplant.es_n 20 Using drones to improve the transplant process.

55. Autonomic media: El Correo Gallego. Date: 20/03/2015. Language: Spanish http://www.elcorreogallego.es/gallego-del-ano/galardones-del-2015/ecg/idNoticia923517/

"Galician of the year" awards nomination for the Dronlife project creators.

56. National media: te interesa. Date: 25/03/2015. Language: Spanish http://www.teinteresa.es/espana/ORGANIZACIONES-TRASPLANTADOS-PROYECTOTRANSPORTAR-ORGANOS_0_1327067966.html

Dronlife at the Transplant National Day acts in Madrid. Summary of the project. "Scientific Labor" Carlos Margarit award.

57. Media: Federación Española de Fibrosis Quística. Date: 25/03/2015. Language: Spanish http://www.fibrosisquistica.org/index. php?pagina $=$ noticias\&esnoticia $=945$ FQ participation on the Transplant National Day acts in Madrid and summary of the acknowledgments given.

58. National media: El economista. Date: 25/03/2015. Language: Spanish 
http://www.eleconomista.es/empresas-finanzas/noticias/6582348/03/15/DIANACIONAL-DEL-TRASPLANTE.html\#.Kku89WLZU9jMgb4

Summary about the National Transplant Day acts in Madrid and the acknowledgments given.

59. National media: Estrategias de inversión. Date: 25/03/2015. Language: Spanish http://www.estrategiasdeinversion.com/invertir-largo/noticias/dia-nacional-deltrasplante-268930

Summary about the National Transplant Day acts in Madrid and the acknowledgments given.

60. Autonomic media: El Correo Gallego. Date: 28/03/2015. Language: Spanish http://www.elcorreogallego.es/galicia/ecg/creadoras-dron-salvavidas-coronangallegas-ano/idEdicion-2015-03-28/idNoticia-923517/

The Dronlife project creators are named "Galician of the year"

61. National media: Fármaco Salud. Date: 09/04/2015. Language: Spanish http://farmacosalud.com/drones-nueva-solucion-para-transportar-organos-entrasplantes-y-vacunas/

Summary about the Dronlife project.

62. Local media: Diario de Ferrol. Date: 07/05/2015. Language: Spanish http://www.diariodeferrol.com/articulo/ferrol/eudi-reconoce-talento-y-logrosalumnos/20150506233832122739.html

Red Nosé Awards, Eudi recognition of their students talent, including the creators of the project Dronlife.

63. Autonomic media: La Voz de Galicia. Date: 01/06/2015. Language: Spanish http://www.lavozdegalicia.es/noticia/galicia/2015/06/01/traslado-organosexploracion-petrolifera-objetivos-galicia/0003_201506G1P7993.htm Drones initiatives in Galicia.

64. Autonomic media: Blog Mundo-R. Date: 10/06/2015. Language: Spanish/Galician 
http://blog.mundo-r.com/es/xente/dronlife

Project Dronlife: their creators and participation in The Drones For Good Award.

65. Media: The UAE Drones for Good Award. Date: 13/07/2015. Language: English https://www.dronesforgood.ae/media/dronlife-making-organ-delivery-transplanteasier-1

Summary about the Dronlife project.

66. Autonomic media: Galicia Ártabra. Date: 14/10/2015. Language: Spanish http://www.galiciaartabradigital.com/archivos/121834 "Galician of the year" awards, where the Dronlife project creators were awarded.

67. Autonomic media: El Correo Gallego. Date: 21/10/2015. Language: Spanish http://www.elcorreogallego.es/tendencias/ecg/1-500-invitados-asistiran-mananaentrega-premios-gallegos-ano/idEdicion-2015-10-21/idNoticia-959293/ "Galician of the year" awards presentation, where the Dronlife project creators were awarded.

68. Autonomic media: El Correo Gallego. Date: 22/10/2015. Language: Spanish http://www.elcorreogallego.es/galicia/ecg/feijoo-presidente-asomase-todos-dias-aoseu-pais-dende-balcon-grupo-correo-gallego-levantou-hai-138-anos/idEdicion-201510-22/idNoticia- 959627/

"Galician of the year" awards presentation, where the Dronlife project creators were awarded.

69. Autonomic media: Galicia hoxe. Date: 23/10/2015. Language: Spanish http://www.galiciahoxe.com/fotos/gh/xxvi-premios-gallegos-anogalardonados/idGaleria-9006/idlmagen-2/ "Galician of the year" awards, where the Dronlife project creators were awarded.

70. Autonomic media: El Correo Gallego. Date: 23/10/2015. Language: Spanish http://www.elcorreogallego.es/gallego-del-ano/gala-de-entrega-de-premios- 
2015/ecg/jovenes-deben-luchar-quieren-porque-hay-futuro-tierra/idEdicion-2015-1023/idNoticia-959704/

"Galician of the year" awards, where the Dronlife project creators were awarded.

71. Autonomic media: Diario de Pontevedra. Date: 26/10/2015. Language: Spanish http://diariodepontevedra.galiciae.com/gl/opinion/456714/de-nuevo-hacia-el-futuro Summary about the Dronlife project.

72. Media: Real Sociedad Matemática Española. Date: October 2015. Language: Spanish http://www.rsme.es/content/view/1856/101/

"Galician of the year" awards, where the Dronlife project creators were awarded.

73. International media: Info Dron. Date: 19/01/2016. Language: Spanish http://infodron.es/id/2016/01/19/opinion-vision-comercial.php Summary about the Dronlife project.

74. International media: Newsweek. Date: 25/01/2016. Language: English http://www.newsweek.com/india-organ-transplant-drones-419013?rx=us Dronlife project to improve the transplant process.

75. Local media: Ferrol 360. Date: 29/01/2016. Language: Spanish http://www.ferrol360.es/el-dron-creado-en-diseno-industrial-para-trasladar-organospara-trasplantes-se-exhibe-en-madrid/ Dronlife participates in the International robotics fair Global Robot Expo

76. Autonomic media: La Voz de Galicia. Date: 29/01/2016. Language: Spanish http://www.lavozdegalicia.es/noticia/ferrol/ferrol/2016/01/29/india-interesa-drontransportador-organos-estudiantes-ferrolanas/00031454089337124139353.htm Dronlife participates in the International robotics fair Global Robot Expo

77. Local media: Diario de Ferrol. Date: 30/01/2016. Language: Spanish http://www.diariodeferrol.com/articulo/ferrol/dron-salvavidas-creado-ferrol-feriaglobal-robot/20160129220305144963.html Dronlife participates in the International robotics fair Global Robot Expo 
78. Mexican media: Diario Puntual. Date: 04/02/2016. Language: Spanish http://www.diariopuntual.com/salud/2016/02/04/12737

Dronlife project to improve transplant process.

79. Mexican media: Transporte.MX. Date: 08/02/2016. Language: Spanish http://www.transporte.mx/drones-para-transportar-organos/ Dronlife project to improve transplant process.

80. National media: Uno más uno. Date: 12/02/2016. Language: Spanish http://www.unomasuno.com.mx/organos-humanos-seran-transportados-por-drones/ Summary about Dronlife and its project.

81. National media: Economía Digital. Date: 07/03/2016. Language: Spanish http://www.economiadigital.es/gles/notices/2015/03/galicia-una-potencia-en-lafabricacion-de-drones-59741.php UAV's sector in Galicia. Dronlife is one of the existing companies.

82. Media: Xunta de Galicia_Igualdade. Date: 08/03/2016. Language: Spanish/Galician http://igualdade.xunta.gal/es/actualidad/feijoo-adianta-que-este-ano-xunta-aprobarapor-primeira-vez-un-programa-de-insercion-0

The Dronlife project creators participate in the official commemoration acts of the Women's Day, organized by the Xunta de Galicia.

83. National media: 20 Minutos. Date: 08/03/2016. Language: Spanish http://www.20minutos.es/noticia/2692468/0/feijo0-anuncia-programa-insercionlaboral-para-maltratadas-acto-que-dio-voz-doce-gallegas-transgresoras/ The Dronlife project creators participate in the official commemoration acts of the Women's Day, organized by the Xunta de Galicia.

84. Autonomic media: Galicia 24 horas. Date: 08/03/2016. Language: Spanish http://galicia24horas.es/2016/03/la-xunta-aprobara-un-programa-de-insercionlaboral-para-mujeres-victimas-de-violencia-de-genero/ 
The Dronlife project creators participate in the official commemoration acts of the Women's Day, organized by the Xunta de Galicia.

85. National media: ABC. Date: 09/03/2016. Language: Spanish

http://www.abc.es/espana/galicia/abci-galicia-escribe-femenino201603091117_noticia.html

The Dronlife project creators participate in the official commemoration acts of the Women's Day, organized by the Xunta de Galicia.

86. Autonomic media: Galicia Confidencial. Date: 09/03/2016. Language: Galician http://www.galiciaconfidencial.com/noticia/28330-xunta-tivo-catro-baixas-listatraballadoras-homenaxeadas-dia-internacional-muller The Dronlife project creators participate in the official commemoration acts of the Women's Day, organized by the Xunta de Galicia.

87. National media: El referente. Date: 03/04/2016. Language: Spanish.

http://www.elreferente.es/robotica/drones-espana-fabricantes-eventos-formacion29521

Drones manufacturers in Spain.

88. American media: Metro. Date: 08/04/2016. Language: English.

http://www.metro.us/lifestyle/medical-robotics-the-future-of-treatment/zsJpdh--XXgisfuq751uY/

Technological advances in the medical field, with references to Dronlife

89. Peruvian media: Metro. Date: 19/04/2016. Language: Spanish. https://issuu.com/metro_peru/docs/20160419_pe_lima

Page 12_Technological advances in the medical field, with references to Dronlife

90. International media: Newday International Media Agency. Date: 22/04/2016.

Language: English.

http://www.newday.mk/flying-heart-by-transport-drone/

Summary about Dronlife. Interests on New Delhi. 
91. Russian media: Metro. Date: 26/04/2016. Language: Russian. https://issuu.com/metro_russia/docs/20160426_ru_petersburg Page 16_Technological advances in the medical field, with references to Dronlife 92. International media: MIT Technology Review. Date: May 2016. Language: German. PDF Summary about the Dronlife project.

93. National media: Proyecta 56. Date: No date registered. Language: Spanish. http://www.proyecta56.com/dronlife/ Dronlife project. Innovation to save lives. 